\title{
MEDICIÓN DE LA CALIDAD UNIVERSITARIA EN CHILE: LA INFLUENCIA DE LOS RANKINGS
}

\section{Claudia Reyes ${ }^{1}$}

\begin{abstract}
RESUMEN
Este artículo se enfoca en el análisis del efecto performativo de los rankings en la calidad universitaria, dando cuenta de los principales resultados de una investigación cualitativa que indaga en una muestra de universidades chilenas. Con una mirada sociológica, se presenta un análisis crítico de los rankings universitarios desde el punto de vista de su construcción y divulgación, asimilándolo a la presencia de un fact-totem y develando el modelo de calidad que suscriben. Se describen los efectos que provocan los rankings en las instituciones y cómo opera el modelamiento de la idea de calidad actualmente vigente, a través de una red de agenciamiento de la que son parte junto con otros, como los medios de comunicación y representantes de las propias universidades a quienes se les asigna un rol performativo. Finalmente, siguiendo la metáfora de imaginar la universidad ideal, se identifican aspectos excluidos de la noción de calidad vigente que se plantean como un nuevo referente, relevándose elementos como la idea de comunidad, mejor docencia y pertinencia de la investigación, entre otros.
\end{abstract}

Palabras clave: agenciamiento sociotécnico, calidad universitaria, fact-totem, performatividad, rankings universitarios.

\section{UNIVERSITY QUALITY MEASUREMENT IN CHILE: THE INFLUENCE OF RANKINGS}

ABSTRACT

This article focuses on the analysis of performative effects of rankings on university quality, showing the main results of a qualitative study on a sample of Chilean universities. From a sociological perspective and assimilating the presence of a fact-totem, a critical analysis of university rankings is presented in regards to its production and release, showing the subscribed quality model. The article describes the effects the rankings have on institutions and how the modeling of the current concept of quality operates, through an agency network which they are part of, along with the media and representatives of the participating universities, whom are ascribed a performative role. Finally, following the metaphor of imagining the ideal university, elements excluded from the current definition of university quality are identified and offered as a new reference, considering aspects such as the idea of community, improved teaching, and relevant research among others.

Keywords: fact-totem, performativity, university quality, sociotechnical, agency, university rankings.

1 Universidad de Concepción, Santiago, Chile. Contacto: creyes@udecsantiago.cl 


\section{Introducción}

La noción de calidad aparece como un concepto central en muchos estudios internacionales acerca del desarrollo de la educación superior de los últimos 35 años y, al igual que en el mundo, en Chile ha sido un tema de debate desde que comenzó a ser parte de la política pública, cobrando gran influencia en la construcción del sistema de educación superior chileno. Hoy la noción de calidad está siendo muy utilizada en el discurso público, especialmente en torno a una reforma al sistema de educación superior que el gobierno actual ha declarado como una de sus principales tareas.

Mucho se ha hablado en los últimos años de la calidad en educación superior, pero sin hacer explícito a qué se refieren los actores con esta idea que aparece claramente naturalizada en sus discursos.

Es por ello que se ha buscado develar las ideas ocultas y preconcepciones que existen en torno a esta noción, tarea abordada a partir de la medición, una práctica común de las ciencias económicas que se ha utilizado crecientemente también en el campo de la educación.

La noción de calidad universitaria se ha instalado en el mundo (y también en Chile) por medio de diferentes sistemas de evaluación y medición que la operacionalizan a través de criterios e indicadores respecto de ciertos aspectos de la universidad, aquellos más relevantes y significativos para quien evalúa. Una de las maneras en que los gobiernos, como agentes reguladores y garantes de la calidad de la oferta educativa, se vinculan con las instituciones de educación superior es a través de estos mecanismos de evaluación y medición de la calidad, entre los cuales se ubican los sistemas de aseguramiento de la calidad, algunos mecanismos de financiamiento estatal (varios de ellos vinculados con los desempeños de las instituciones), y los rankings universitarios, aunque estos últimos, pese a que operan bajo las mismas lógicas de medición, son desarrollados mayoritariamente por entidades no estatales.

A juicio de algunos expertos nacionales, los sistemas de medición de la calidad buscan entregar a los estudiantes la información 
necesaria para disminuir las asimetrías de información al momento de elegir una carrera o institución donde cursar estudios superiores (Bernasconi, 2006; Meller y Rappoport, 2006), aunque en realidad no se sabe si efectivamente esta información es utilizada y considerada una ayuda por parte de los postulantes.

La elección de indicadores y estándares que se utilizan en las mediciones, y la forma en que estos se manejan, parecen remitir a una idea global de calidad. Esta idea de calidad universitaria, o de cómo debe ser una buena universidad, proveniente de la institucionalidad vigente, no es explícita y parece encarnar en la actualidad un discurso dominante que está influyendo de alguna manera en el funcionamiento de las instituciones universitarias, especialmente porque se vincula con poderosos incentivos. Se sospecha que las instituciones responden a los requerimientos de calidad de la institucionalidad vigente de una manera "adecuada y funcional", movidas por fuerzas que no necesariamente reflejan las creencias e ideas que abrigan las comunidades al interior de estas instituciones.

Se estudiaron los rankings universitarios para develar cuál es la idea de calidad universitaria que sustentan y cómo esta noción está influyendo en el funcionamiento de las universidades chilenas. Se cree que los rankings contribuyen de alguna manera a instalar una idea de calidad a la cual las universidades responden sistemáticamente a través de una cierta conducta o comportamiento que cumple con el modelo de calidad esperado. Luego, la pregunta que orientó la investigación buscó develar la forma en que los rankings influyen en las universidades chilenas. Específicamente, cómo contribuyen a moldear una idea de calidad de las universidades en Chile.

Para responder a esta pregunta, el artículo recogió los principales resultados de una investigación empírica desarrollada para este fin, donde destaca un análisis crítico acerca de los rankings universitarios desde el punto de vista de su construcción y divulgación, y cómo estos influyen en las instituciones universitarias, produciendo ciertos efectos. El análisis se desarrolló bajo un marco teórico que puso en juego dos conceptos fundamentales y relativamente recientes en las 
ciencias sociales: el enfoque de la performatividad y la noción de fact-totem.

\section{Marco teórico}

La performatividad es un concepto que nace en los años sesenta bajo la teoría del lenguaje (Austin, 1976; Searle, 1997) y desde allí ha sido extensamente tratado por la sociología (Ariztía, 2012; Butler, 2007; Callon, 2007; Foucault, 2009; Mackenzie, Muniesa \& Siu, 2007; Ramos, 2012a). Por otro lado, el concepto de fact-totem (De Santos, 2009) -o también llamado de estadísticas públicas-, es un dispositivo que provee cifras que se han hecho parte de la vida cotidiana, influenciando las conductas y despertando gran interés en estudios sociológicos de los últimos años (Davis, Fisher, Kingsbury \& Merry, 2012; Espeland \& Sauder, 2007; Morley, 2001; Morley \& Aynsley, 2007; Prèvost \& Beaud, 2012).

Las estadísticas representan cosas diferentes según el contexto en que se presenten; mientras para los expertos pueden ser simples datos o información, para un público no experto pueden significar visiones muy diferentes que se hacen posibles a través de la creencia o doxa (Bourdieu, 2007; Bourdieu \& Eagleton, 1997), dejando así de ser meros antecedentes y transformándose en representaciones colectivas. A partir del estudio referido a la estadística "riesgo país"2 en la época previa al corralito ${ }^{3}$, el sociólogo argentino Martín de Santos elaboró el concepto de fact-totem, definiéndolo como una estadística con elevada visibilidad mediática y pública que se articula con relatos identitarios centrales de una colectividad. En los medios, "las estadísticas parecen ser inmunes contra la opinión: parcelas de la realidad misma presentadas en forma prístina como noticias" (De Santos, 2009, pág. 172).

2 Índice financiero que mide el precio de los bonos según se negocian en los mercados internacionales secundarios

3 El corralito se denominó a la restricción impuesta por el gobierno argentino a la de la libre disposición de dinero en efectivo de plazos fijos, cuentas corrientes y cajas de ahorros entre el mes de diciembre de 2001 y el 2 de diciembre de 2002, fecha en la que se permitió la liberación de los depósitos retenidos. 
Para comprender mejor, se puede reflexionar respecto de las propias estadísticas nacionales, aquellas que acompañan cotidianamente y que se han hecho parte de las vidas de los ciudadanos. Nótese cómo se reacciona ante estadísticas de uso común en la medicina actual, donde las narrativas que sustentan la existencia de graves enfermedades se apoya fuertemente en cifras de prevalencia: por ejemplo, número de muertes por cáncer de pulmón incentivan acciones para dejar de fumar o estadísticas de cáncer de mamas llevan a las mujeres a realizarse exámenes y controles médicos periódicos para prevenir dichas enfermedades. A los ciudadanos les preocupan las estadísticas del crimen y los delitos, afectando las opiniones y conductas en relación con la seguridad pública, cómo protegerse de robos y asaltos, y cómo estas cifras contribuyen al desarrollo del lucrativo mercado de los seguros.

En el enfoque de la performatividad, las investigaciones más conocidas se han desarrollado en el campo de las ciencias económicas (Callon, 1998; Callon, 2007; Mackenzie, 2007; Mackenzie, Muniesa, \& Siu, 2007), sin dejar de mencionar el aporte del investigador chileno Claudio Ramos al estudio de la performatividad de las ciencias sociales (Ramos, 2012a; Ramos, 2012b; Ramos, 2014; Ramos, 2015). Para Callon y MacKenzie, "la ciencia económica, más que constituir una mera observación de su objeto, se hace parte de tal objeto y contribuye a su configuración; de este modo los mercados son así, tanto objetos como producto de investigación" (Ramos, 2014, p. 159). Para el autor, la performatividad de las ciencias económicas se refiere a la instalación de un modelo, pero "no se trata de un modelo que logre aprehender el mundo, sino de cómo el mundo termina actuando de la manera en que el modelo lo predice a través de sus fórmulas" (Ramos, 2012a, p. 221). En estos estudios, básicamente se distingue el hecho de que las ciencias no solo dan cuenta de representaciones que constatan la realidad, sino que al mismo tiempo la comunicación social de la que son parte les agrega un potencial performativo, tanto dentro del campo científico como fuera de él (Ramos, 2012b). La idea de pobreza (Ramos, 2015) y el bullying son ejemplos de relatos científicos que, acompañados de la construcción de datos, permiten modelar la realidad en contextos que están fuera del campo científico. 
Según Ramos (2012b, p. 225), "la performatividad es modelamiento, a través de cadenas de reiteraciones, pero es un modelamiento que va variando a través del proceso y que es inherentemente inestable". Las ideas de la ciencia que moldean el mundo real no son transferidas meramente por la lectura de papers científicos, requieren de un agenciamiento que se articula, traduce e instala a través de procesos diferentes y recurrentes que incluyen dispositivos y personas, conversaciones, formas de cálculo y discursos que van modelando gradualmente las conductas que el modelo predice. A este proceso se le ha denominado agenciamiento sociotécnico (Callon, 2007, p. 320).

Ahora bien, para que un fact-totem exista, así como para que un hecho o discurso científico sea performativo, deben darse ciertas condiciones de posibilidad donde convergen tres ámbitos discursivos comunes que, en conjunto, incrementan su poder de representación y capacidad performativa: la existencia de los números y las matemáticas que tienen por sí solos un poder de representación objetiva e impersonal que para algunos tienen mayor grado de credibilidad que las palabras (De Santos, 2009, p. 171); la existencia del discurso científico que, con su aura de verdad, goza de una alta confianza pública (Bourdieu, 1984; Brunner, 2014; De Santos, 2009; Espeland \& Sauder, 2007; Ramos, 2012a); y el discurso de los medios de comunicación, que permite una amplia circulación y acceso a las ideas a un público masivo y que se caracteriza por una narrativa que habla de historias de ascenso y caídas (De Santos, 2009; Ramos, 2012b).

La estructura cuantitativa de los rankings universitarios se sustenta en las mismas lógicas de construcción del conocimiento científico que las estadísticas públicas, haciéndolos aparecer como un fact-totem y, al mismo tiempo, como un dispositivo con capacidad performativa. La potencia de ambos conceptos y su vinculación en un mismo dispositivo los transforma en una herramienta especial para analizar la calidad universitaria como objeto de estudio. 
164 MEDICIÓN DE LA CALIDAD UNIVERSITARIA EN CHILE: LA INFLUENCIA DE LOS RANKINGS - C. Reyes

\section{Marco metodológico}

La investigación realizada se enmarcó en un estudio de tipo cualitativo basado principalmente en las técnicas de entrevista en profundidad y análisis del discurso en torno a textos y documentos de uso público. El trabajo de campo consistió en describir, desde la perspectiva de los sujetos, las nociones que tienen respecto de los mecanismos de medición de la calidad universitaria y, en particular, acerca de la construcción y usos otorgados a los rankings universitarios. Se indagó en los aspectos metodológicos y en el uso de indicadores como representaciones válidas de una idea de calidad universitaria, así como también, en la respuesta de las universidades a estos mecanismos de medición y al uso que ellas hacen de los resultados de los rankings con otros fines. Considerando que la palabra calidad está cargada de significados, se utilizó la idea de "imaginar una universidad ideal" como metáfora de la calidad, pero sin hablar de ella (Barnett, 2013).

Se realizaron entrevistas semiestructuradas utilizando una pauta común, a dos grupos de informantes, representantes de una muestra de diez universidades ubicadas a lo largo del país: un grupo de seis rectores y cinco expertos(as) en calidad (una de estas últimas especialistas es consultora externa y no está adscrita a ninguna institución). Para ambos grupos de entrevistados se diseñó una adecuada distribución de los informantes, considerando su pertenencia a diferentes categorías de universidades, utilizando la tipología que identifica cuatro grupos diferentes de universidades: docentes, docentes con proyección en investigación, de investigación y doctorado en áreas selectivas, y de investigación y doctorado (Reyes $\&$ Rosso, 2013); y la tipología de instituciones estatales, tradicionales y privadas. Además, se consideró una equilibrada distribución geográfica de las universidades participantes a lo largo del país, distinguiendo entre universidades regionales y metropolitanas; y se intentó equilibrar la proporción de hombres y mujeres entre los entrevistados.

Dadas estas definiciones muestrales, es necesario destacar que no se logró encontrar en los resultados diferencias significativas entre las opiniones de los entrevistados según las categorías consideradas. 
Una explicación posible es el escaso tamaño de la muestra, cuestión que debiera considerarse como hipótesis en futuros trabajos de investigación. De acuerdo con los resultados obtenidos, lo que se pudo constatar es que frente a los temas de calidad no se presentan grandes discrepancias entre las opiniones de los representantes de las universidades chilenas.

Una segunda forma de recolección de información se basó en textos nacionales e internacionales publicados en medios de comunicación web o impresos donde se aplicó la técnica de análisis del discurso y de contenidos como principal método de trabajo. Se intentó describir y analizar críticamente los discursos que subyacen tras las publicaciones de los rankings universitarios a través de tres casos concretos de estudio (Ranking QS Latinoamérica, Ranking Revista Qué Pasa y Ranking GEA Universitas-El Mercurio) que fueron elegidos conforme a los siguientes criterios: que tuvieran más de un año de existencia o que hubieran sido publicados más de una vez; que incluyeran una cantidad significativa de universidades chilenas; y que tuvieran un impacto mediático significativo o que aparecieran como noticia en medios nacionales. Se excluyeron del estudio otros rankings internacionales que no incorporaban universidades chilenas significativamente, pues para analizar los efectos al interior de éstas se estimó necesario que las instituciones consideradas en el estudio aparecieran en ellos.

Además, se trabajó con una selección de noticias asociadas a los rankings publicados en distintos formatos y medios de comunicación chilenos. Fundamentalmente, en esta parte se trabajó con el análisis de contenidos y del discurso a fin de describir y analizar en profundidad las narrativas que proponen los rankings.

\section{Principales resultados de la investigación}

\subsection{Análisis crítico de los rankings universitarios}

Para hablar de los rankings y de su influencia es necesario previamente conocer qué es un ranking, cómo y en qué contexto se construye y describir las principales características que lo hacen una narrativa 
referida a la calidad universitaria. Para ello, estos fueron analizados desde dos perspectivas diferentes: su producción o construcción y su divulgación.

\section{a. Producción o construcción de los rankings}

Los rankings se refieren a un ordenamiento de las universidades, de mayor a menor valor, según un puntaje calculado a partir de un algoritmo que se construye sobre la base de la ponderación de indicadores o índices que representan diferentes dimensiones de calidad de las universidades. Entre estos índices se puede incluir, además, un puntaje asociado al resultado de encuestas de percepción con una determinada ponderación. Se trata, en principio, de la configuración de un modelo matemático que involucra el uso de ciertas variables ya conocidas. Su validez tiene que ver con una lógica matemática y coherencia metodológica que representa la abstracción de una idea de calidad universitaria a partir de la información disponible. Este desarrollo se circunscribe, en la lógica de los expertos o especialistas que lo construyen, a un ejercicio científico cuyo resultado quedará validado si la metodología utilizada es consistente en el tiempo y no tiene cuestionamientos lógico-matemáticos.

El algoritmo de cálculo que representa la calidad (C) para una universidad (i) puede escribirse como:

$$
\mathrm{C}_{\mathrm{i}}=\sum_{j=1}^{m}\left(\sum_{k=1}^{l}(I * \beta)_{k} * \propto\right)_{j}
$$

Donde $i=1, n$ según el número de instituciones a evaluar; $j=1, m$ según el número de dimensiones a medir; $\alpha_{j}$ es el porcentaje con el que se ponderan las dimensiones a evaluar. Además, $0 \leq \alpha \leq 1 ;(I * \beta)_{k}$, corresponde al indicador I ponderado en un porcentaje $\beta$, donde $0 \leq \beta \leq ; y k=1, l$ según el número de indicadores que representa cada dimensión.

El ejercicio de quienes producen los rankings representa un esfuerzo por reducir la complejidad de una idea, cuyo abordaje matemático lo permite, hasta llegar a un número y posición que se hace fácilmente transmisible a un público masivo. En esta traducción de la calidad a un lenguaje matemático se tiende a discriminar y 
elegir solo ciertas dimensiones y parámetros de la calidad a partir de los datos que están disponibles y que han sido aparentemente validados por fuentes oficiales. Se podría decir que, como en todo enfoque positivista, para dar solución a un problema hay un esfuerzo por reducir la complejidad de la realidad observada, donde la calidad quedaría representada solo por ciertos aspectos, aquellos más significativos para quienes construyen los rankings. Con este argumento no se quiere minimizar el valor del método científico y de las matemáticas, cuestión que parecería absurda considerando la larga historia de avances en el conocimiento que esta ciencia ha hecho posible, sino simplemente destacar el punto donde la ciencia -en este caso las matemáticas-, aborda la complejidad de una idea en el proceso de objetivación de dimensiones eminentemente cualitativas.

Por ahora se destaca que, así como en las estadísticas públicas y en los discursos con capacidad performativa, la representación de la calidad de una determinada universidad en lenguaje matemático también parece determinante para legitimar los rankings en el contexto de su construcción.

Ahora bien, respecto de la metodología, indicadores y datos, explícitamente reconocidos como un mecanismo de medición, los entrevistados expresaron fuertes críticas a la manera en que los rankings miden la calidad, fundamentalmente por la forma en que estos han sido construidos. A los cuestionamientos más o menos conocidos en la literatura en relación con la metodología empleada y uso de algunos indicadores (Albach, 2012; Marginson, 2007; Marginson \& Van der Wende, 2007; Meller \& Rappoport, 2006; Morley \& Aynsley, 2007; Ordorika \& Rodríguez, 2010; Usher \& Savino, 2006), se agregan discrepancias en torno a aspectos más específicos como el uso de ciertos indicadores para medir calidad de la docencia. Sin embargo, también se reportaron algunos aspectos positivamente valorados de los rankings, como la transparencia que aportan y la posibilidad de comparar instituciones.

Los rankings aparecen bajo la sospecha de los entrevistados. La mayoría de ellos, conocedores de la realidad universitaria, mira con suspicacia el resultado que arrojan, enfatizando la necesidad de 
una adecuada lectura conjunta de los indicadores, pues conocen los detalles de su construcción. Son conscientes de las dificultades que conlleva la cuantificación de dimensiones cualitativas de las funciones que desarrolla la universidad y por tanto expresan -como expertoslas precauciones necesarias para una adecuada comprensión de los resultados. Algunos de los entrevistados los caracterizaron como una fotografía del conjunto de universidades en un determinado momento, otros, como simple información. Uno de los entrevistados se refiere a los rankings en los siguientes términos:

[los encuentro] extremadamente peligrosos por la simplificación. [El ranking] por esencia, tiende a numerar lo que es cualitativo. ¿Y cómo mides la calidad de una sonrisa? Si [los rankings] están construidos en base a indicadores, entonces son sesgados. [Los indicadores] están llenos, llenos, llenos de trampas; y si [yo] no soy consciente del sesgo, puedo producir injusticias brutales. (Rector universidad privada, de investigación y doctorado en áreas selectivas, metropolitana).

La mayoría de los entrevistados repara en la selección de ciertos indicadores y en la forma arbitraria en que se ponderan para llegar a un resultado agregado. Los entrevistados saben que los rankings están dirigidos a un público no conocedor de la realidad universitaria y son cautos en advertir que si bien aportan información acerca de las universidades a quienes no conocen del tema, constituyen solo una posible lectura de la calidad universitaria que debe asumirse con espíritu crítico, como un discurso que en ningún caso debe tomarse como una verdad absoluta.

Todos los rankings evalúan fundamentalmente la calidad de los estudiantes y su proceso formativo, así como también de los académicos, la investigación y la gestión de las instituciones; para lo cual se basan en el uso de indicadores o índices agregados que miden tales dimensiones, aunque ponderados en diferente proporción. Así, figuran indicadores de amplio uso tales como: estudiantes por profesor, porcentaje de académicos con grado de doctor, publicaciones por académico, impacto de las publicaciones, porcentaje de académicos y estudiantes internacionales, tasa de retención y titulación, y encuestas de percepción de empleabilidad y prestigio de las instituciones. Las 
ponderaciones se asignan en forma aparentemente arbitraria, pues en ninguno de los casos estudiados ni en otros en los que se investigó este aspecto, explican en su metodología los fundamentos bajo los cuales se definen las ponderaciones de las dimensiones e índices evaluados.

Otro aspecto que puede destacarse es el tipo de indicadores utilizados. El uso de indicadores de eficiencia y productividad parecen haberse trasplantado desde la empresa a la organización universitaria como una forma de medir calidad. La eficiencia y productividad aplicada a la universidad se plantea como una suerte de medida del rendimiento de los académicos. ¿Cuánto es capaz de producir o de hacer un académico en un período de tiempo? Por ejemplo, "número de publicaciones científicas anuales por académico", es un indicador de amplio uso a nivel mundial y nacional que representa bastante bien la idea de productividad. La dimensión temporal asociada al desempeño humano permite identificar estándares del tipo: ¿cuál es la cantidad máxima de publicaciones que puede generar en un año un investigador? Es posible identificar también los indicadores denominados de eficiencia docente (o gestión docente) que intentan ser un sustituto válido de la calidad de la docencia. Estos miden relaciones de desempeño en torno a dicha labor o, de manera agregada, en torno a la institución: la tasa de retención estudiantil representa, entonces, la capacidad de los profesores y/o de la institución para mantener a sus estudiantes y evitar su deserción, pero nada dice respecto de qué capacidades debe tener ese profesor. Del mismo modo, la tasa de estudiantes por profesor o duración real de las carreras son indicadores que (siguiendo la metáfora empresarial), en cierta forma hacen mirar la universidad como una organización para la "producción eficiente de profesionales".

Uno de los indicadores que presentó reparos por parte de algunos entrevistados y que se utiliza en algunos rankings construidos en Chile (Qué Pasa, América y Economía, GEA UniversitasEl Mercurio), es el que se refiere a los recursos asignados a las universidades por concepto de Aporte Fiscal Indirecto, AFI. Utilizar solo el criterio PSU para definir cuál universidad es de mejor calidad es una cuestión ampliamente discutible. ¿Es mejor aquella que logra captar a los mejores estudiantes (representados por los mayores 
puntajes PSU)? O, dada la baja selectividad de las universidades chilenas actuales, ¿aquella que logra transformar en profesionales a estudiantes que ingresan con carencias formativas (y baja PSU)? La relatividad del concepto "calidad de estudiante" bajo este indicador hace cuestionable la forma en que algunos rankings abordan esta dimensión.

El uso de encuestas a los empleadores donde se pretende evaluar la calidad de los graduados es un indicador que también merece mayor reflexión según algunos de los entrevistados. Se mide el valor agregado entregado por la universidad a sus graduados que, además, deben ser sujetos empleables en el mercado del trabajo, sin considerar que la empleabilidad depende de varios otros factores, en cierta medida exógenos a la institución, como por ejemplo, la oferta de empleo en el país o el capital social del que dispone el graduado y su capacidad de articular redes que faciliten su acceso al mercado del trabajo, cuestiones de las cuales la universidad no se hace cargo. De acuerdo con los rankings que usan este tipo de encuestas, la calidad de la universidad quedaría entonces determinada por la capacidad de la institución para formar profesionales empleables en el mercado laboral.

Los rankings replican indicadores utilizados internacionalmente para validar su performance nacional. Esto es así en la mayoría de los índices utilizados para medir resultados en investigación (productividad e impacto), aunque ciertamente en este caso, porque el mecanismo que se utiliza para validar y medir lo que es o no un aporte al conocimiento, opera a nivel mundial y las bases de datos donde se indexan las publicaciones científicas válidas son de acceso internacional (ISI Web of Science, Scopus). Varios de los indicadores de investigación son usados también en las evaluaciones de agencias acreditadoras y por los gobiernos cuando miden desempeño de las universidades, señalando con esto que la diversificación de su uso por parte de distintos actores en el sistema universitario mundial y nacional avala su uso como medida válida de una idea de calidad de la investigación aparentemente institucionalizada en el mundo (Marginson, 2007; 2009). 
Un elemento fundamental sobre el cual no se ha puesto demasiada atención en los diseños metodológicos de los rankings es la confiabilidad de los datos con que se cuenta para el cálculo de indicadores. En general, los rankings apelan a la confianza depositada en las organizaciones que emiten datos, creyendo que por ser fuentes públicas y oficiales, institucionalizadas en los gobiernos, resultan confiables, pero siendo exhaustivos, no hay información explícita en estas agencias acerca de la confiabilidad de la información que producen. Este punto de vista pone un gran manto de duda sobre la validez de los resultados obtenidos y resulta crucial al momento de analizar la metodología de los rankings.

Reforzando el argumento anterior, la mayoría de los entrevistados conoce los artilugios que se pueden usar para llegar a un buen resultado en las posiciones finales de los rankings $y$, por eso, varios de ellos cuestionaron tajantemente el origen de los datos que se utilizan en las mediciones, aunque estos son entregados directamente al Ministerio de Educación de Chile, por las propias instituciones; hay sospechas de que la información entregada no refleja cabalmente la realidad, sino que ha sido alterada para mejorar los resultados de los indicadores y, por tanto, la posición final en los rankings. Algunos entrevistados cuestionaron no tanto el sistema de medición en sí, sino la calidad y confiabilidad de la información que lo alimenta. Uno de ellos lo expresa del siguiente modo:

La seriedad de los datos también puede tener algún efecto [en los rankings]. ¿La institución entrega el dato real, objetivo o entrega aquello que le conviene entregar? Por eso te digo que tengo algunas aprensiones con eso, pero no dejo de desconocer que gran parte de esos datos, son datos oficiales que nosotros mismos hemos entregado al Ministerio [y] que ellos han constatado. (Experto, universidad privada, docente con proyección en investigación, metropolitana).

b. Divulgación: después de la publicación de los rankings Una nueva perspectiva para mirar con detención los mecanismos utilizados por los rankings es la etapa de su divulgación. Un enfoque que sale del ámbito de su construcción e involucra a nuevos actores, ya no a quienes los construyen, sino fundamentalmente a los medios 
de comunicación y a las propias universidades, quienes serán los responsables de amplificar o intensificar el discurso propuesto por los rankings.

La etapa de divulgación se inicia cada año una vez publicado el ranking. Todos disponen de su propio sitio web donde despliegan los resultados y una diversidad de información que incluye aspectos metodológicos e información acerca de sus autores. Los rankings gozan de una amplia difusión mediática que se concentra en el período de tiempo inmediatamente a continuación de su primera publicación y su alcance mediático considera la aparición destacada como noticia nacional en los medios de prensa, radio y televisión (24 Horas, 2014; El Mercurio, 2012; La Tercera, 2014); la inclusión en páginas de publicidad universitaria en diarios y revistas, así como la difusión en los sitios web de las propias universidades (P. Universidad Católica de Chile, 2014; Universidad Andrés Bello, 2015a; Universidad de Talca, 2014).

Si se observa más detenidamente la relación entre los rankings y los medios de comunicación es posible constatar una fuerte vinculación que va más allá de una mutua validación: los medios traducen el discurso de calidad de los rankings y lo sacan del contexto centrado en la construcción, donde se prioriza el lenguaje científico, orientándolo a una audiencia masiva que se ubica en el contexto de lo social y lo público. Los medios amplifican el discurso de los rankings o, como dice Ramos, dan cuenta de la intensidad del discurso (Ramos, 2012b) y le permiten llegar ampliamente a los públicos que le interesan, legitimando de este modo el poder de su discurso.

Los medios de comunicación intervienen en la forma en que se transmite la información, minimizando en cierta medida la complejidad de la medición y de una idea de calidad multidimensional que ahora se expresa en forma parcial y simplificada, aportando otra manera de leerlos. Los medios realizan una suerte de traducción del lenguaje científico hacia uno más coloquial y comprensible por un público masivo, donde lo relevante ahora son otros aspectos, diferentes a las cuestiones metodológicas y conceptuales, como por ejemplo quiénes ocupan los primeros y los últimos lugares en 
el ordenamiento de posiciones. Una revisión de los titulares de los medios para referirse a las noticias sobre los rankings describe un lenguaje melodramático (De Santos, 2009), caracterizado por los cambios de posición de las universidades rankeadas, quiénes suben y quiénes bajan, cuáles son las que mejoran y cuáles las que empeoran su calidad (P. Universidad Católica de Chile, 2011; P. Universidad Católica de Valparaíso, 2014; Universidad de Concepción, 2014; Universidad Diego Portales, 2014; Universidad de los Andes, 2014).

\section{c. El lenguaje de los rankings y la creencia}

El discurso de los rankings en su forma más simple y básica, expresado a través de los medios de comunicación, es una noción de calidad universitaria asociada a la posición que una universidad ocupa en un determinado ordenamiento. De este modo, se establece en el público no conocedor una idea de mejor y peor calidad, asociando a las universidades que se ubican en los primeros lugares de las tablas y que, consecuentemente, obtienen los puntajes más altos, como aquellas de mejor calidad y, análogamente, identificando a las que se ubican en los últimos lugares de las tablas con los puntajes más bajos, como aquellas de peor calidad.

La simpleza de este juicio en términos de su legibilidad y fácil comprensión, más la confianza otorgada a quien lo emite, parecen generar en la opinión del público "no experto" una idea de calidad que permanece en el tiempo y que tiende a instalarse en el imaginario nacional, afectando ciertamente en forma positiva o negativa el prestigio de las universidades o, en otras palabras, su capacidad para ser percibidas como universidades de buena calidad. La autoridad histórica del lenguaje matemático y del método científico utilizado en la construcción de los rankings contribuye a legitimar su existencia, aportando verdad al discurso que proponen y facilitando la creencia (Callon, 1998; De Santos, 2009; Espeland \& Sauder, 2007; Ramos, 2012a). Cuando actúa la creencia (Bourdieu, 2007) se omite la reflexión y una comprensión más profunda en torno a la construcción de los rankings; lo que aparece como un ejercicio técnico para sus creadores o para quienes conocen el mundo universitario, una fotografía o simple información de las instituciones, no es vista de igual modo por el público "no experto". Por el contrario, el juicio de 
calidad parece instalarse en la opinión pública como una sentencia permanente.

Por otra parte, la cotidianeidad con que se viven las estadísticas públicas y la fuerza de verdad que ellas traen consigo permite también mirar los indicadores y datos que utilizan los rankings (en su construcción), como una estructura amparada en la representación de objetividad que tienen por sí solos los números. Siguiendo a De Santos, es posible asimilar los rankings a la presencia de un fact-totem:

... un símbolo poderoso que condensa la atención social y suscita respuestas apasionadas de vastas audiencias; en ellos coinciden periódicamente los medios de comunicación y el público y son un punto de encuentro colectivo pues traspasan a una sociedad consumista de información (De Santos, 2009, p. 148).

Los rankings enuncian un discurso fáctico, un discurso que crea realidad en torno a la idea de calidad universitaria.

El lenguaje de los rankings impacta directamente en la forma en que se instala la idea final de calidad universitaria. Dos de los entrevistados se refieren a la manera en que esto ocurre, señalando que en los rankings la gente se queda solo con un número o posición, con el valor final:

Yo creo que es [información] muy engañosa si no se tiene mucho cuidado... Hay que enseñar a la gente a leerla. Si yo no sé leer, [entonces] leo desarticulado completo y la mayoría de la gente se queda con la fracción final, [con el resultado]. (Rector, universidad privada, de investigación y doctorado en áreas selectivas, metropolitana).

Los rankings también tienden a generar segregación; lo que hacen es que distorsionan en cómo te identifican en la opinión pública, en quiénes están interesados en investigar en las universidades y en quiénes asignan los recursos. La gente se mueve por el lugar del ranking: "ah, no, es que esta está más arriba”, pero no se pregunta porque está ahí. (Rector, universidad tradicional, docente con proyección en investigación, regional). 
Para ilustrar mejor este argumento es preciso volver al marco teórico. En los estudios de la performatividad de las ciencias económicas, Callon dice que "para predecir el comportamiento de los agentes, la teoría económica no debe ser verdadera, solo necesita ser creída por todos, incluso, si las creencias no tienen relación con el mundo, el mundo termina pareciéndosele, o siendo la creencia" (Callon, 2007, p. 322).

\subsection{La idea de universidad que esconden los rankings}

Si se observa con detención qué es lo que se mide, cuáles son los énfasis que ponen los rankings en la definición y ponderación de dimensiones e indicadores, se revela un cierto modelo de universidad que será el referente. Este se encuentra bajo el paradigma de las grandes universidades de investigación. De acuerdo con este argumento, la universidad de mejor calidad sería: aquella de mayor tamaño que realiza las funciones de docencia y que también desarrolla intensivamente la investigación; la que hace más y mejor investigación en toda la variedad de disciplinas existentes; aquella que dispone de un cuerpo académico mayoritariamente con grado de doctor o PhD. (lo que supuestamente hace a la institución más productiva en publicaciones indexadas y en patentes de invención); y es también la institución que logra el mayor impacto mundial de esas publicaciones. Este modelo de universidad en Chile es también el que presenta los mejores indicadores de eficiencia docente, aquella institución donde los estudiantes no desertan y se titulan en el menor tiempo posible, donde los graduados mayoritariamente encuentran trabajo poco tiempo después del egreso, obteniendo las más altas remuneraciones. Es decir, una universidad integrada por un perfil de estudiante "ideal" o más propiamente dicho, imaginario o inexistente.

De acuerdo con este paradigma comparativo, validado mundialmente en los principales rankings universitarios publicados (Shanghai University Rankings, 2014; Times Higher Education, 2014), las instituciones de menor tamaño y aquellas concentradas en áreas disciplinarias focalizadas aparecen en una situación desfavorable. En el caso chileno, también quedarían al margen las universidades 
que se declaran solo como universidades docentes o con baja labor en investigación.

La inamovilidad o aparición reiterada, año a año, de las universidades intensivas en investigación en los primeros lugares de los rankings, parece deberse entonces a cierta superioridad que logran estas universidades por acercarse más al modelo de universidad propuesto. Un modelo que parece trascender, pues se revela no solo en los actuales rankings universitarios internacionales y nacionales, sino también en las mediciones que contemplan otros procesos como la acreditación o aquellas que miden desempeño universitario para la asignación de fondos públicos. Uno de los entrevistados se refirió a este tema del siguiente modo:

El sistema de medición de indicadores que tenemos tiene un paradigma que lo concibe. La mejor [universidad] es aquella donde los recursos se reparten a la mejor ciencia, a los mejores científicos, con las mejores ideas, ahí van las becas a los doctorados, los proyectos Conicyt, todas las cosas, o todo lo que son fondos concursables para... la calidad, son para complejidad. (Rector, universidad estatal, de investigación y doctorado en áreas selectivas, regional).

La situación de Chile no deja de parecerse a la del resto del mundo, ya que los referentes universitarios son internacionales, como lo expresa uno de los entrevistados: "A nosotros aquí [en Chile] nos ponen los criterios de calidad que nos impone el mundo desarrollado, porque estamos en un mundo globalizado" (Rector, universidad privada, de investigación y doctorado en áreas selectivas, metropolitana). En el resto del mundo, la idea de mejor universidad, aquella con la cual hay una comparación implícita, es también la gran universidad de investigación, la que aparece en los primeros lugares de los rankings más conocidos. La superioridad de las universidades norteamericanas es manifiesta en los resultados y el modelo de Universidad de Harvard es probablemente el que mejor encarna las aspiraciones del mundo globalizado.

Pese a que la mayoría de los entrevistados compartió la opinión de que los rankings adolecen de otras dimensiones de calidad tanto 
o más relevantes que las que actualmente se evalúan, casi todos validaron los indicadores que actualmente se utilizan para medir la investigación, pues esta representaría una parte esencial y distintiva de la misión universitaria. Uno de los entrevistados expresa esta idea de la siguiente manera:

Jamás se me pasaría por la mente decir que no es relevante, como decíamos antes, por ejemplo, variables absolutamente cuantitativas como los papers que produce, la calidad de las revistas donde publican los papers, el número de proyectos de investigación que tiene, las visitas o interacciones que tiene con otras universidades de buena calidad a nivel internacional; es evidente que todas esas cosas son medibles y son variables muy importantes, eso está fuera de discusión... como piso tiene que haber una tasa de artículos de investigación en las revistas de más alto nivel por académico, mínima que tiene que estar. Ahí yo no hago ninguna relativización... y si [la universidad] no tiene ninguna [publicación] es porque está muy, muy mal... y no debería llamarse universidad. (Rector, universidad estatal, de investigación y doctorado, metropolitana).

\subsection{Cómo opera la performatividad de los rankings universitarios}

Como la pregunta de investigación lo sugiere, no es solo la existencia de los rankings universitarios lo que permite alcanzar los niveles de modelamiento logrados, sino que junto con ellos se han reconocido otros mecanismos contribuyentes a la instalación de la idea de calidad que actualmente se tiene. Reconociendo esta situación, se abordará de una forma más genérica los efectos de medir (Espeland \& Sauder, 2007), anticipando la presencia de otras agencias que conforman una red que realiza el efecto performador.

¿Qué ocurre al interior de las universidades con la aparición pública de los rankings? ¿Cómo se manejan internamente los resultados y qué acciones toman las instituciones? Las consecuencias de medir la calidad de las instituciones produce en las propias universidades ciertos efectos, algunas veces adversos, otras beneficiosos, que fueron reconocidos por varios de los entrevistados. 


\section{a. La respuesta de las instituciones}

Entre los entrevistados, la mayoría reconoce que los rankings tienen mayor importancia que la que declaran públicamente, especialmente las autoridades universitarias, quienes habitualmente minimizan su impacto. Se reconoce que el resultado de un ranking, sea este positivo o negativo para la institución, moviliza internamente a las comunidades y se discute en torno al resultado logrado. Uno de los entrevistados lo expresa del siguiente modo:

Yo creo que [en la universidad] le ponen mucha atención, en general. Tengo la impresión de que es un tema importante, porque se preocupan. Cuando les va bien, se lo cuentan a medio mundo, lo publican o lo hablan, o se sienten felices y cuando les va mal, encuentran que el ranking es malo... Toda institución, aunque no lo reconozca, yo creo que sí debe dar una miradita a ver dónde quedó, si mejor o peor que el año pasado... porque sabe que los demás la están viendo a través de eso. A lo mejor, a veces sienten que es injusto, hay muchas instituciones, me imagino, que sienten que es injusto porque quisieran mostrar, a lo mejor otro lado, otras cosas que se están haciendo, o que se están esforzando por hacer mejor. (Consultora experta, metropolitana).

b. La competencia que generan los resultados de las mediciones entre las instituciones

Una respuesta evidente de las universidades frente a los rankings se refleja en la competencia que generan. Este sentido de competencia entre las instituciones por lograr cada vez un mejor resultado es reconocida por los entrevistados, contribuyendo a este efecto el diseño mismo del mecanismo de medición, que es con fines comparativos. Solo aquellas que están en las mejores posiciones serán destacadas en las noticias, tendrán mayor publicidad y serán reconocidas por la opinión pública como universidades de prestigio por su calidad. Se trata, entonces, de esforzarse internamente y de seguir las estrategias necesarias para ir con el tiempo ganando posiciones en los rankings. Uno de los entrevistados reflexiona sobre este punto del siguiente modo:

No sé cuán importantes son los rankings y esta locura por los rankings... La reificación de los rankings que tienen que 
ver un poquito con esta ideología neoliberal, también de la competencia, de que tenemos que medirnos y yo soy mejor que usted porque yo me saqué 180 y usted se sacó 169. (Rector, universidad estatal, de investigación y doctorado, metropolitana).

La necesidad de aparecer en los rankings y de ocupar posiciones destacadas revela que ellas asumen la propuesta de calidad que los rankings proponen, apareciendo así una primera manifestación de la noción de performatividad. Al asumir las universidades la propuesta de calidad de los rankings, aceptan también que las dimensiones de calidad que se evalúan y los indicadores que se utilizan son los que efectivamente describen la calidad universitaria, la respuesta positiva de sumarse al ranking y trabajar para aparecer en él es un actuar conforme a las reglas del juego que pone el ranking, favoreciendo el modelo de calidad vigente.

\section{c. Estrategias para mostrar lo bueno que somos}

Pareciera que la misma actitud estratégica para "pasar la prueba" que han desarrollado las instituciones frente a la acreditación, ocurre frente a los rankings. Las universidades desarrollan mecanismos para lograr un mejor puntaje y responder exitosamente, acercándose a los estándares que los rankings están poniendo, para responder al modelo de universidad que se está pidiendo. Algunos de los mecanismos utilizados para alterar los indicadores son expuestos por el siguiente entrevistado:

Hay formas [de mejorar los indicadores]... Cuando uno mide retención, uno cambia el reglamento de estudio y puede mejorar el indicador. En el tiempo de titulación, si todos [los estudiantes] hacen una permanencia en el extranjero, se atrasa el tiempo de titulación. (Rector, universidad estatal, de investigación y doctorado en áreas selectivas, regional).

La metáfora utilizada por otro entrevistado resume claramente el manejo de estrategias para mejorar los indicadores y así aparecer mejor posicionado en una medición:

Cuando hablo de medición de calidad, uno comienza a jugar con el indicador. Hay mucho, mucho, mucho del niñito que 
no quiere ir a clases, entonces agarra el termómetro y lo pone en el guatero y se lo muestra a la mamá. (Rector, universidad estatal, de investigación y doctorado, metropolitana).

El juego de los indicadores para mostrar mejores resultados es una clara evidencia de cómo se modela la noción de calidad en las universidades, entonces el efecto performativo parece haberse logrado: las universidades han asumido el juego y responden de la manera esperada. Cuando un enunciado es performativo, hay una respuesta esperada de antemano -dice Foucault (2009)-, las universidades asumirán esa idea de calidad y no otra y responderán lo que se espera de ellas; es decir, el comportamiento producido por el discurso en la realidad responde, efectivamente a lo predicho, a los criterios descriptivos del modelo (de la ciencia) (Callon, 2007).

\section{d. El uso de los rankings para fines publicitarios}

Como ya se ha dicho, una característica distintiva de los rankings es que se publican y difunden a través de medios de comunicación masivos. Revistas, periódicos y sitios web son los medios predilectos que permiten el consumo masivo de esta información sobre calidad universitaria por parte de un público no conocedor del tema. Pero no solo los medios de comunicación contribuyen a este fin con las noticias en torno a la aparición de los rankings, las universidades también han asumido la tarea de replicar y difundir sus resultados, aportando de este modo a la asimilación de la noción de calidad que se ha descrito y se constituyen como una nueva entidad dentro de una red agencial que comienza a configurarse (Callon, 2007, pp. 319-320).

Aunque varios de los entrevistados reconocen las limitaciones de los rankings y son cautos en su lectura, una de las consecuencias de su existencia es que las universidades los utilizan con fines publicitarios. En aquellos casos donde conviene, señalan algunos, los rankings contribuyen a fortalecer la tarea de marketing que hacen las casas de estudio, ayudando a posicionar en el imaginario público la calidad lograda por la institución en el último ranking publicado, siempre y cuando la universidad haya obtenido una posición destacada como resultado. En otras palabras, pese a que muchas instituciones critican la manera en que estos miden calidad y cuestionan sus resultados, los 
validan, promoviéndolos como noticia en los distintos formatos de publicidad que utiliza la institución. Sobre las acciones que toman las universidades frente a los rankings donde aparecen bien posicionadas, uno de los entrevistados señala:

La respuesta [de las universidades] es la misma que hacen los colegios con el famoso Simce $u$ otras pruebas estandarizadas, que en el fondo usan [el ranking] como una herramienta de marketing... son estrategias. (Rector, universidad tradicional, docente con proyección en investigación, regional).

\section{e. La medición produce una distorsión del quehacer de las universidades}

Se reconoce que cualquier medición que produce como resultado un ordenamiento de posiciones permitirá a las instituciones manipular los resultados, pues es posible anticiparse para "pasar la prueba". En la opinión de varios entrevistados, las universidades trabajan para obtener el mejor resultado, para lograr más puntos, muchas veces desviándose de su verdadera misión. Varios ejemplos avalan este argumento: la preparación de los colegios para la PSU, las escuelas de medicina frente al Eunacom (Examen Único Nacional de Conocimientos de Medicina) y los colegios frente al Simce, son evidencias de que el impacto de las mediciones sobre las instituciones existe. Hay un aprendizaje de las instituciones a lo largo del tiempo orientado a ser exitoso en el logro de una posición y, de este modo, la institución se hace funcional al resultado del ranking, es decir, comienza a actuar en función del resultado. La siguiente cita de una de las entrevistas refleja bien este punto:

Esto [los rankings] se ha prestado para que en vez de preocuparse de la calidad o de la pertinencia de la investigación, reconociendo la importancia que se le reconoce a esa variable en muchas partes, hace que la universidad se centre solo en aquello que le da los puntos que necesita, y eso produce una distorsión del quehacer de la universidad. (Rector, universidad estatal, de investigación y doctorado, metropolitana).

Varios entrevistados reconocen que se trabaja para mejorar los indicadores y que muchas veces esto produce problemas, pues 
se dejan de hacer esfuerzos importantes en otros aspectos que son parte del quehacer universitario. Hoy día lo que miden los rankings o las mediciones de la calidad en general, marcan la pauta de las cosas que son realmente importantes para decir si una universidad es o no de una cierta calidad, dejando fuera aquello que no se mide y que muchas veces responde a la verdadera misión de la institución. Una entrevistada, señala sobre este punto:

De alguna forma los [los rankings] van guiando un poco también hacia dónde va la cosa. Las instituciones no son indiferentes a cómo van guiando los rankings... [van guiando] una idea de universidad, una idea de qué cosas son importantes. Entonces claro, si se incluye el número de doctores, de profesores... son las cosas importantes que se miden. (Experta consultora, metropolitana).

Una segunda evidencia acerca de la función performativa de los rankings es que señalan un camino que indica qué es lo importante de medir para dar cuenta de la calidad de la universidad. Actúan agenciando la noción de calidad que se intenta institucionalizar y, de este modo, se presentan como parte de la red de agenciamiento sociotécnico de la que nos habla Callon (2007), son una tecnología, un medio de agenciamiento de la idea de calidad que representa el discurso dominante, que deja fuera varios aspectos que, al no considerarse, son subvalorados o parecieran inexistentes.

\section{f. Se genera un efecto negativo en la docencia}

Uno de los efectos destacados por los entrevistados es el reconocimiento de que habría una tendencia a subvalorar la docencia como parte de las funciones universitarias prioritarias. La docencia se distingue como una dimensión difícil de medir y escasamente abordada por los rankings, en oposición al valor que se le otorga actualmente a la investigación como parte fundamental en cualquiera de los mecanismos de medición de calidad universitaria. Uno de los entrevistados se refiere a este punto de la siguiente manera:

Sin duda que a quienes gestionamos instituciones de educación superior, los rankings nos producen unos efectos, en el sentido de que para mejorar el ranking hay siempre que ir generando 
mejores condiciones, por ejemplo, para los investigadores que son los que dan puntos para el ranking. Y no todas las universidades son instituciones de investigación (quizás las más grandes, las más complejas pueden darse ese lujo), pero universidades comunes y corrientes, las universidades "de a pie", digamos, tienen que combinar labores docentes... [Entonces], hay un efecto en la docencia que es perverso. (Rector, universidad estatal, de investigación y doctorado en áreas selectivas, regional).

La mayoría de los rankings internacionales no tiene en consideración adecuadamente la docencia, minimizando con ello la importancia de la calidad del proceso de enseñanza-aprendizaje en la formación de los estudiantes. Y aquellos rankings que sí dan cuenta de ella, se basan en la medición de indicadores que no reflejan adecuadamente la calidad del proyecto educativo de la institución. Este efecto negativo en la docencia queda en evidencia porque la preocupación actual de las instituciones tiende a ser solo sobre aquellos aspectos que le otorgan visibilidad, puntos para aparecer mejor posicionado, donde esta dimensión no aparece efectivamente evaluada, sino aproximada a través de indicadores que representan medidas de eficiencia de procesos docentes (estudiantes por académico, tasa de retención o tasa de titulación, entre otros). La opinión de uno de los entrevistados sobre este tema es la siguiente:

Yo creo que no están los indicadores de la calidad de la docencia [adecuados], no están bien... Sin duda que el cuerpo de profesores es importante, pero supongo que puede estar dedicado a investigación... Entonces [los indicadores docentes] no dan cuenta de la calidad del proceso educativo, [solo se] ven... resultados. Por ejemplo, si uno mirara estos colegios de la educación media que hacen dos o tres cursos en uno..., son lo más eficientes, todos aprueban, pero si hay calidad ahí, me provoca serias dudas... Debiéramos buscar mecanismos [para] evaluar de una mejor forma la calidad de la docencia. (Rector, universidad estatal, de investigación y doctorado en áreas selectivas, regional).

Se han identificado varias de las respuestas de las universidades como los efectos performativos de los rankings, entre ellas, cómo las instituciones juegan para obtener los mejores resultados y las más 
altas posiciones, playing to the test (Espeland \& Sauder, 2007, p. 2), evidenciando el modelamiento de un discurso de calidad que se ha instaurado a través del tiempo como el discurso dominante que actúa subrepticiamente en las propias universidades. Es la manifestación de la profecía autocumplida (Callon, 2007, p. 321), la constatación de la respuesta esperada de parte de las instituciones.

Para Callon (2007, p. 316) un discurso es performativo cuando contribuye a construir la realidad que está describiendo y, en este caso, es posible constatar que el discurso de calidad produce, en la realidad, la noción de calidad que está describiendo, una noción reconocida por los indicadores estandarizados que se utilizan para medirla. Los rankings aparecen entonces como un mecanismo agencial, son un medio que muestra esta operación.

\subsection{Iterabilidad o capacidad de acción del discurso performativo}

Michel Callon articuló la noción de agenciamiento sociotécnico para representar de este modo una red de mecanismos, procedimientos y personas que hacen posible la asimilación de las ideas que viajan desde un contexto científico hacia afuera de esos dominios (Mackenzie, Muniesa, \& Siu, 2007). La existencia de una cadena agencial que sostiene el discurso sobre calidad muestra que es una narrativa coconstruida que se repite a través de diferentes actores y dispositivos, no de la misma manera dice Butler (1990), sino aportando a la construcción del relato sobre calidad que, así como en los discursos de género, "incluyendo a los propios actores, ha venido a creer y a actuar como creencia" (Butler, 1990, p. 297).

El uso reiterado de los mismos indicadores y datos en diferentes tipos de mediciones de la calidad, o en mediciones realizadas con distintos fines, incluidos otros rankings, como en los procesos de acreditación, en evaluaciones de programas y proyectos, en mediciones del desempeño para la asignación de recursos públicos, su uso reiterado en seminarios y conferencias presentados por asesores y expertos, representa lo que para Butler es "la repetición estilizada de los actos en el tiempo" (1990, p. 297), la repetición del mismo discurso 
de calidad bajo diferentes agentes, que en conjunto contribuyen a incrementar la capacidad de agenciamiento de la idea.

En la perspectiva de esta red agencial, los rankings universitarios pasan a ser una tecnología o dispositivo agencial, una interfaz mediadora del discurso performativo de calidad que se logra a través del tiempo, apoyado a su vez en la cadena agencial que repite y potencia el discurso.

Por otra parte, en medio de este viaje desde el dominio de las ciencias, hay otros actores que surgieron en la investigación y que se identifican como parte de la red, pues tienen la capacidad de rearticular el discurso de los rankings, utilizando un lenguaje reconocible. Entre ellos destacan los medios de comunicación y los rectores entrevistados como representantes de las instituciones universitarias.

Los medios constituyen una parte fundamental en la estructura agencial del discurso, en la manera en que Callon lo describe y, son tal vez una finalidad para los rankings, pues en ellos descansa gran parte de su capacidad performativa. Como ya se ha señalado, su poder de difusión alcanza a un público masivo y permite mantener en el tiempo el discurso sobre calidad propuesto, multiplicándolo a través de sus propias redes mediáticas.

\section{a. El rol performativo de los rectores}

En el grupo de autoridades entrevistadas se reveló cierta falta de libertad (que el cargo conlleva) para explayarse y abordar con sinceridad las opiniones que guardan las personas más allá de sus cargos. Varias veces, algunos entrevistados se remitieron a una respuesta "adecuada", o bien, "políticamente correcta", para no comprometer opiniones que se contravienen con la identidad o etiqueta que el cargo les impone. Uno de los entrevistados se refirió explícitamente a esta situación cuando se le planteó cuál era su idea de una universidad ideal, señalando:

Yo pienso más bien en la microeconomía de la universidad, he tratado de hacer un esfuerzo por buscar la utopía, en oposición 
a [lo que sería] una respuesta de catálogo: que [la universidad] esté súper vinculada al extranjero, que tenga las mejores redes internacionales, que sea capaz de organizar los sistemas... (Rector, universidad estatal, de investigación y doctorado en áreas selectivas, regional).

En esta situación, ciertamente limitante frente a lo que se dice o deja de decir, se pudo reconocer la presencia de la agencia, parte de la cadena agencial que permite performar la idea de calidad actualmente vigente en el país. Las respuestas de catálogo a las que alude la cita anterior son justamente parte de la retórica del discurso dominante acerca de la calidad universitaria que se ha venido instalado institucionalmente, discurso que asumen en forma natural y casi inconscientemente algunos de los rectores entrevistados, y que es fácilmente identificable a través de indicadores de desempeño estandarizados, reconocibles y medibles. Sin que se les preguntara por ello, inevitablemente los entrevistados destacaron con orgullo, en más de una oportunidad, hechos, datos e indicadores (contratación de académicos con doctorado, aumento de publicaciones indexadas, entre otros) realizados por sus propias instituciones como ejemplos de calidad o de lo bien que lo están haciendo como universidad, mostrando aquellas dimensiones en que la universidad representada tiene evidencia de buenos resultados y ciertamente el interés en que se pongan de relieve. La siguiente cita de una de las entrevistas muestra bien esta cuestión:

Te lo digo yo como rector, nosotros que lo hemos definido como universidad, hoy día tenemos un 91\% de los programas acreditados, todas las ingenierías por cuatro años y con cuatro agencias diferentes... las decisiones que se toman, se toman siempre pensando en los mismos estándares que la institución se ha ido imponiendo... (Rector, universidad privada, docente, metropolitana).

El enunciado de la cita puede leerse de este otro modo: "te lo digo desde mi estatus (cargo de rector), desde mi autoridad, por lo tanto debes confiar en la sinceridad y verdad de lo que digo: lo estamos haciendo bien como institución, estamos respondiendo bien a lo que se espera de nosotros". Se revela también en esta opinión, que el discurso de calidad que esta autoridad maneja responde a los criterios de calidad esperados, a aquellos que el discurso ha modelado, 
basándose en indicadores y estándares como los mencionados; "tenemos el 91\% de los programas acreditados", es ciertamente un indicador representativo del discurso de calidad dominante.

Sin embargo, la actitud de los entrevistados fue cambiando a lo largo de la entrevista, oscilando por momentos hacia las respuestas de catálogo, es decir, momentos donde aparece claramente la agencia, y momentos donde aparece la parrhesia, un hablar franco y veraz de la persona que se arriesga a emitir su opinión (Foucault, 2009). Atención especial merece una pregunta donde se logran percibir bastante bien estos cambios, permitiendo a los entrevistados mayor soltura y comodidad en la entrevista que se apreció no solo en un discurso más reflexivo y en divagar hasta construir una idea, sino también en ciertos gestos corporales. La pregunta por "imaginar una universidad ideal" otorgó libertad al entrevistado, fue una invitación a crear un mundo, a hablar de la utopía que los posicionó frente a un futuro por inventar, sacándolos de sus actuales posiciones y preocupaciones. En este caso, en varias de las entrevistas, la agencia performadora no se reveló y, por el contrario, se dieron a conocer ciertas dimensiones de la calidad universitaria que quedan excluidas del discurso dominante actual.

\section{Dimensiones excluidas del discurso actual de calidad}

Las ideas marginadas del discurso actual son las que los entrevistados rescatan al imaginar cómo sería la universidad ideal. La pregunta por imaginar la universidad (Barnett, 2013), usada como recurso epistemológico, permite al sujeto soñar y pensar en la utopía, pero en una utopía viable de nueva universidad. Según Barnett, el ejercicio de imaginar pone al sujeto en la situación de pensar algo que no existe en el mundo real, pero que se le parece, pues se construye a partir de ideas previas y de los déficits percibidos en el imaginario actual.

Aunque no faltaron las respuestas de catálogo o políticamente correctas, los entrevistados describieron cómo podría ser esta institución ideal que, en varios aspectos, contrasta con el paradigma comparativo actual de universidad. 


\subsection{Diversidad del alumnado}

Para algunos entrevistados, un rasgo distintivo en la universidad ideal debe ser la diversidad del alumnado, pues esta condición contribuye a un mejor aprendizaje, no solo de conocimientos, sino de aspectos valóricos y cívicos que fueron considerados de importancia dentro de la formación profesional. Una universidad inclusiva que permita aprender a vivir en tolerancia y aceptación del otro es una de las miradas que concibe a la universidad como un reflejo del país.

\subsection{Una comunidad de aprendizaje}

La mayoría de los entrevistados se refirió a la universidad como una comunidad que involucra a los distintos estamentos universitarios, especialmente a profesores y alumnos en el proceso de enseñanza aprendizaje. El desafío está, entonces, en formar esas comunidades que parecen haberse perdido en el tiempo, según uno de los entrevistados, siendo uno de los pilares de la universidad desde sus inicios. Hoy en día la tecnología ha ocupado espacios que fomentan el autoaprendizaje, lo que ha repercutido en la relación entre docentes y estudiantes, la cual se percibe lejana de lo que fuera tiempo atrás.

\subsection{Mejor docencia}

Un elemento esencial en la universidad ideal se refiere a la calidad de la docencia, a la necesidad de mejores profesores para el pregrado. Los entrevistados apelan a que la universidad ideal necesita de maestros, personas comprometidas con la docencia que sepan enseñar, nutriéndose de la investigación en torno a las propias prácticas educativas.

\subsection{Investigación y pertinencia}

Se reconoce la importancia de la investigación en la universidad ideal, pero también se reclama por la pertinencia de esa investigación. Esta pertinencia es entendida como la respuesta a una adecuada vinculación con el medio donde la universidad está situada. La responsabilidad de la universidad ideal, para muchos de los entrevistados, se sitúa en una función pública, lo que significa hacerse parte en la solución de los problemas del país y del entorno local donde la universidad 
está ubicada, en su comunidad o región, contribuyendo al desarrollo económico, social y cultural de su entorno. Varios de los entrevistados fueron enfáticos al señalar la pertinencia como rasgo distintivo de la universidad ideal, para salvarla de transformarse en un centro de estudios avanzados donde solo prima la productividad en investigación de punta o bajo estándares internacionales.

\subsection{Gestión universitaria}

Los temas asociados al liderazgo, a la forma de gobernar y a la organización universitaria no estuvieron fuera de la idea de universidad ideal. Se develaron atributos como el liderazgo de los equipos directivos, el sistema de gobierno universitario, la necesaria representación de todos los estamentos universitarios y un gobierno corporativo que respeta los principios de la autonomía como condición estructural del sistema universitario. La existencia de estos elementos en la universidad ideal contribuirá -según los entrevistados- a una mejor gestión de la institución.

\section{Conclusiones y discusión final}

La calidad universitaria se revela a través de los rankings con un estatuto cosificado que deviene de la operación que transforma aspectos cualitativos de la universidad en cifras. Amparados en la verdad de la ciencia y en la objetividad del lenguaje matemático, los rankings logran reducir a un número la calidad de una cierta universidad y presentarla en un ordenamiento de posiciones donde es posible comparar sus resultados con otras. De este modo, usando los indicadores y los datos adecuados, los rankings entregan información acerca de las universidades a un público no conocedor del tema y, apoyados por los medios de comunicación, facilitan su llegada a una audiencia masiva. Es en este contexto donde interviene el discurso fáctico de los rankings, que opera a través de la creencia, cuando las personas son persuadidas a creer y aceptar como verdad una idea de calidad universitaria que en realidad no conocen.

Al interior de las instituciones universitarias los rankings producen ciertos efectos como la competencia por demostrar lo bien que lo están haciendo. Siguiendo las reglas del juego (playing to the 
test), las universidades se esfuerzan por mejorar sus resultados en aquellas dimensiones e indicadores que dan puntos para el ranking. Así, todos los esfuerzos estarán puestos en responder al modelo, descuidando otras áreas, aun cuando estas pueden representar aspectos fundamentales del quehacer universitario, como la docencia, que en la opinión de muchos entrevistados ha quedado rezagada, produciéndose una distorsión en el quehacer de las instituciones. El modelo de mejor universidad que promueven los rankings subvalora dimensiones que quedan excluidas de la idea de calidad.

En el estudio se ha constatado que el discurso de calidad de los rankings contribuye a producir en la realidad la noción de calidad que se está describiendo, una noción que se reconoce en el uso de indicadores estandarizados que se utilizan para medirla. Bajo esta premisa, los rankings se presentan como un dispositivo de agenciamiento sociotécnico (Callon, 2007) dentro de una suerte de metadiscurso de la calidad que sostienen distintos actores, prácticas, conversaciones y entidades que operan en el país como agentes del modelo de calidad que se ha institucionalizado, o que está en un proceso continuo de modelamiento. Así, los rankings realizan una intervención dentro de una compleja y permanente gestión de agenciamiento del discurso dominante de calidad en el país.

Distintas prácticas de medición y cálculo que utilizan los mismos indicadores que los rankings se reconocen, por ejemplo, en los procesos de acreditación y en la asignación de financiamiento público a las instituciones (fondos por desempeño). Tales mecanismos, junto con otros actores, como los productores de rankings, medios de comunicación, miembros de las propias instituciones, como expertos y asesores, o las autoridades que fueron parte en esta investigación; y también espacios de conversación como seminarios y congresos integran, entre otros, la red de agenciamiento sociotécnico de la calidad.

A partir de las dimensiones e indicadores evaluados, el énfasis de los rankings está puesto principalmente en los resultados de investigación que las universidades son capaces de lograr, subordinando otras posibles dimensiones de calidad a un segundo 
plano, y mostrando de este modo que hay un modelo oculto, no explícito de universidad que los rankings ponen como referente a través de lo que están midiendo. Se trata de la gran universidad de investigación, modelo al que pocas de las instituciones nacionales pueden acercarse; aquella que logra los mejores resultados en los rankings mostrando una permanente superioridad frente al resto, solo porque puede dar mejor cumplimiento a los indicadores que miden los rankings.

El modelo que estos suscriben converge con la idea de universidad de clase mundial (Salmi, 2009), el cual destaca la investigación como uno de sus componentes esenciales, junto con la captación de los mejores talentos del mundo dentro de sus académicos y estudiantes, una adecuada gestión y gobernanza y, la disponibilidad de suficientes recursos monetarios para su financiamiento. Corresponde al modelo de universidad actualmente vigente que sitúa a las universidades en el mundo, abriendo las fronteras de los países para que compitan globalmente por el liderazgo, lo que se ha visto en grandes campañas de internacionalización de la educación superior de países como Estados Unidos y Australia, cuyos resultados se visibilizan en los rankings como evidencia de calidad y reconocimiento para los gobiernos sobre sus sistemas de educación superior (Douglass, 2016; Marginson, 2007).

Con esta investigación emerge la posibilidad de reflexionar acerca de la idea de universidad, pensada desde sí misma, cuestión que ha sido abordada también en recientes investigaciones que, en gran medida, se contraponen al modelo vigente y surgen como vías alternativas a él (Barnett, 2011; Douglass, 2016; Guzmán-Valenzuela, 2016).

Las dimensiones excluidas de la calidad que surgieron en la presente investigación, representan para los entrevistados las funciones que una universidad ideal debe cumplir: la relevancia asignada a la docencia, en contraposición al amplio espacio que hoy se asigna a la investigación; la pertinencia de la investigación, pero no solo de una investigación validada por su impacto internacional, sino que responda también a las necesidades locales de las comunidades 
donde se insertan las instituciones, cumpliendo así el rol público que se le reconoce a la institucionalidad universitaria; y la necesidad de una mejor gestión institucional que involucre un adecuado liderazgo de los equipos que la conducen y una equilibrada gobernanza.

La propuesta de la universidad transformativa (GuzmánValenzuela, 2016) como modelo de nueva universidad pública se aproxima en esta conversación; su autora indaga en el rol público de la universidad, intentando dar una respuesta respecto de cómo esta función se produce, para lo cual propone un nuevo referente que coincide con la presente investigación en el valor que los actores otorgan al rol público de la universidad, como una de las dimensiones relevantes que le da sentido a su existencia. Sin embargo, en su planteamiento asume ciertos supuestos que hacen difícil su realización, pues pareciera que este nuevo modelo de universidad solo tendría cabida en un mundo no neoliberal, cuestión que no profundiza suficientemente. Esto plantea la pregunta acerca del sistema socioeconómico del país donde la universidad existe y la incidencia que esta tiene en la transformación de la nación y sociedad a la que pertenece.

Otras propuestas que aparecen en publicaciones recientes, como el modelo de la flagship university (Douglass, 2016) o la universidad ecológica (Barnett, 2011) suponen también la emergencia de nuevos espacios y modos de interacción de la universidad con su entorno social más local, asumiendo y haciendo explícita la responsabilidad que le cabe para servir a la sociedad en la que se inserta. Son ideas que emergen en distintos lugares del mundo desde conversaciones todavía marginales de actores al interior de las instituciones y por lo mismo, aún carecen de la fuerza necesaria para oponerse a la idea totalizadora del modelo de universidad imperante. En este caso, ¿tendrá la universidad chilena actual la suficiente autonomía, influencia y capacidad para pensarse a sí misma y asumir un camino transformador?

La presente investigación abre un desafío que se suma a los anteriores para conocer más profundamente la idea de universidad ideal como nuevo referente de calidad universitaria, supuesto que 
apenas se conoce y que está presente en los actores que participan del mundo universitario. Indagar en la opinión de estudiantes y académicos, junto con quienes la gestionan, contribuiría a descubrir dimensiones alternativas al discurso performativo actual de calidad universitaria.

\section{Referencias}

Albach, P. (2012). The globalization of college and university rankings. Change, 44(1), 26-31. http://dx.doi.org/10.1080/00091383.2012.636001

Ariztía, T. (2012). Produciendo lo social. Usos de las ciencias sociales en el Chile reciente. Santiago de Chile: Universidad Diego Portales.

Austin, J. (1976). How to do things with words. New York: Oxford University Press.

Barnett, R. (2011). The coming of the ecological university. Oxford Review of Education, 37(4), 439-455. http://dx.doi.org/10.1080/03054985.2 011.595550

Barnett, R. (2013). Imagining the university. New York: Routledge.

Bernasconi, A. (2006). La difícil tarea de clasificar universidades. Rankings y clasificaciones como reacción a la heterogeneidad institucional. Calidad en la Educación, 25, 81-95.

Bourdieu, P. (1984). Homo academicus. Paris: Les Editions de Minuit.

Bourdieu, P. (2007). El sentido práctico. Buenos Aires: Siglo XXI editores.

Bourdieu, P. \& Eagleton, T. (1997). Doxa y vida cotidiana: una entrevista. En T. Eagleton (Ed.), Ideología (pp. 295-308). Barcelona: Paidós.

Brunner, J. J. (2014). Sociología de la sociología. Estudios Públicos, 133, 147 163.

Butler, J. (1990). Actos performativos y constitución del género: un ensayo sobre fenomenología y teoría feminista. En S. E. Case (Ed.), Performing feminisms: Feminist critical theory and theater (pp. 270-282). Baltimore: Johns Hopkins University Press.

Butler, J. (2007). Género en disputa. El feminismo y la subversión de la identidad. Barcelona: Paidós.

Callon, M. (1998). The laws of the markets. Oxford: Blackwell Publishers.

Callon, M. (2007). What does it mean to say that economics is performative? En D. Mackenzie, F. Muniesa, \& L. Siu (Ed.), Do economists make 
markets?: On the performativity of economics (pp. 311-357). Princeton: Princeton University Press.

Davis, K., Fisher, A., Kingsbury, B., \& Merry, S. E. (2012). Governance by indicators. Oxford: Oxford University Press.

De Santos, M. (2009). Los fact-totems y la imaginación estadística: la vida pública de una estadística en la Argentina de 2001. Apuntes de Investigación, 18, 147-180.

Douglass, J. A. (2016). The new flagship university: Changing the paradigm from global rankings to national relevancy. London: Palgrave Macmillan.

El Mercurio. (2012). Prestigioso ranking ubica a cuatro UES chilenas entre las 10 mejores de Latinoamérica. Recuperado de http://www.emol.com/ noticias/nacional/2012/06/13/545415/prestigioso-ranking-ubica-acuatro-ues-chilenas-entre-las-10-mejores-de-latinoamerica.html

Espeland, W. \& Sauder, M. (2007). Rankings and reactivity: How public measures recreate social worlds. American Journal of Sociology, 113(1), 1-40. http://dx.doi.org/10.1086/517897

Foucault, M. (2009). El gobierno de sí y de los otros. Buenos Aires: Fondo de Cultura Económica de Argentina.

Guzmán-Valenzuela, C. (2016). Unfolding the meaning of public(s) in universities: Toward the transformative university. Higher Education, 667-679.

La Tercera. (2014). Ranking QS. Universidad de Chile y PUC destacas entre las mejores 250. Recuperado de http://www.latercera.com/noticia/ nacional/2014/09/680-596145-9-ranking-qs-universidad-de-chile-ypuc-destacan-entre-las-mejores-250.shtml

Mackenzie, D. (2007). Is economics performative? Option theory and construction of derivatives markets. En D. Mackenzie, F. Muniesa, \& L. Siu, Do economists make markets? (pp. 54-86). New Jersey: Princeton University Press.

Mackenzie, D., Muniesa, F., \& Siu, L. (2007). Do economists make markets? On the performativity of economics. Princeton: Princeton University Press.

Marginson, S. (2007). Global university rankings: Implications in general and for Australia. Journal of Higher Education Policy and Management, 29(2), 131-142. http://dx.doi.org/10.1080/13600800701351660

Marginson, S. (2009). Open source knowledge and university rankings. Thesis Eleven, 96(1), 9-39. http://dx.doi.org/10.1177/0725513608099118

Marginson, S. \& Van der Wende, M. (2007). To rank or be ranked: The impact of global rankings in higher education. Journal of 
Studies in International Education, 11(3-4), 306-329. http://dx.doi. org/10.1177/1028315307303544

Meller, P. y Rappoport, D. (2006). Nueva metodología para un ranking de universidades chilenas. Calidad en la Educación, 25, 57-77.

Morley, L. (2001). Producing new workers: Quality, equality and employability in higher education. Quality in Higher Education, 7(2), 131-139. http:// dx.doi.org/10.1080/13538320120060024

Morley, L. \& Aynsley, S. (2007). Employers, quality and standards in higher education: Shared values and vocabularies or elitism and inequalities? Higher Education Quarterly, 61(3), 229-249. http://dx.doi.org/10.1111/ j.1468-2273.2007.00353.x

Ordorika, I. \& Rodríguez, R. (2010). El ranking Times en el mercado del prestigio universitario. Perfiles Educativos, 23(129), 8-29.

P. Universidad Católica de Chile. (2011). P. Universidad Católica de Chile sube al puesto 250 en el ranking QS. Recuperado de http:// relacionesinternacionales.uc.cl/destacados-18

P. Universidad Católica de Chile. (2014). UC se ubica como la mejor universidad de América Latina en el Ranking QS. Recuperado de http://www.uc.cl/ es/component/content/article/15569-uc-se-ubica-como-la-mejoruniversidad-de-america-latina-en-el-ranking-qs

P. Universidad Católica de Valparaíso. (2014). PUCV sube siete lugares en Ranking QS y se ubica en el $29^{\circ}$ puesto a nivel latinoamericano. Recuperado de http://prensa.ucv.cl/?p=33194

Prèvost, J. G. \& Beaud, J. P. (2012). Statistics, public debate and the state, 18001945. London: Pickering \& Chatto (Publichers) Limited.

Ramos, C. (2012a). El ensamblaje de ciencia social y sociedad. Santiago de Chile: Ediciones Universidad Alberto Hurtado.

Ramos, C. (2012b). Investigación científica y performatividad social: el caso del PNUD en Chile. En T. Ariztía (Ed.), Produciendo lo social. Usos de las ciencias sociales en el Chile reciente. Santiago de Chile: Ediciones Universidad Diego Portales.

Ramos, C. (2014). Datos y relatos de la ciencia social como componentes de la producción de realidad social. Convergencia, Revista de Ciencias Sociales, 21(66), 151-177.

Ramos, C. (2015). Poverty as epistemic object of government: State cognitive equipment and social science operations. Social Science Information, 54(1), 91-114. http://dx.doi.org/10.1177/0539018414556178 
Reyes, C. \& Rosso, P. (2013). Una nueva clasificación de las universidades chilenas. En Clasificación de Instituciones de Educación Superior (Vol. 2, pp. 135-151). Santiago de Chile: Educación-ES.

Salmi, J. (2009). El desafio de crear universidades de rango mundial. Washington D.C.: Banco Mundial.

Searle, J. (1997). La construcción de la realidad social. Barcelona: Paidós.

Shanghai University Rankings. (2014). Academic ranking of world universities. Recuperado de http://www.shanghairanking.com/es/ARWUMethodology-2014.html - 2

Times Higher Education. (2014). The world university rankings. Recuperado de http://www.timeshighereducation.co.uk/world-universityrankings/2014-15/world-ranking/methodology

Universidad Andrés Bello. (2015a). Ranking QS ubica la U. Andrés Bello en el top 100 latinoamericano. Recuperado de http://investigacion. unab.cl/noticias/ranking-qs-ubica-la-u-andres-bello-en-el-top-100latinoamericano/

Universidad de Concepción. (2014). UdeC se ubica entre las tres mejores universidades de docencia del país. Recuperado de http://www.udec. cl/panoramaweb2/2014/11/udec-se-ubica-entre-las-tres-mejoresuniversidades-de-docencia-del-pais/

Universidad Diego Portales. (2014). UDP sube 15 puestos en ranking QS 2014. Recuperado de http://www.udp.cl/noticias/detalle_noticia. asp?noticiald $=5823$

Universidad de los Andes. (2014). Uandes subió seis puntos y se ubicó en 9 lugar en ranking de calidad de El Mercurio-Universitas. Recuperado de http:// www.uandes.cl/noticias/uandes-subio-seis-puestos-y-se-ubico-en-9lugar-en-ranking-de-calidad-de-el-mercurio-y-universitas.html

Universidad de Talca. (2014). Universidad destaca entre las cuatro mejores en calidad docente. Recuperado de http://www.utalca.cl/link.cgi// SalaPrensa/admision/8515

Usher, A. \& Savino, M. (2006). Estudio global de los rankings universitarios. Calidad de la Educación, 25, 33-53.

Recibido: 17/12/ 2015

Aceptado. 31/05/2016 